\title{
Ethenolysis of renewable methyl oleate catalyzed by readily accessible supported group VI oxo catalysts".
}

Pascal Rouge, ${ }^{\mathrm{a}}$ Yassine Bouhoute, ${ }^{\mathrm{a}}$ Nicolas Merle, ${ }^{\mathrm{b}}$ Kai C. Szeto, ${ }^{\mathrm{a}}$ Aimery De Mallmann, ${ }^{\mathrm{a}}$ Laurent Delevoye, ${ }^{\mathrm{b}}$ Régis M. Gauvin, ${ }^{\mathrm{c},{ }^{*}}$ Mostafa Taoufik. ${ }^{\mathrm{a},{ }^{*}}$

a Université de Lyon, Univ. Lyon 1, CPE Lyon, CNRS UMR 5265, Laboratoire de Chimie Catalyse Polymères et Procédés (C2P2), Bat 308F, 43 Bd du 11 Novembre 1918, F-69616

Villeurbanne, France. E-mail : mostafa.taoufik@univ-lyon1.fr

b Univ. Lille, CNRS, Centrale Lille, ENSCL, Univ. Artois, UMR 8181 - UCCS - Unité de Catalyse et Chimie du Solide, F-59000 Lille, France

${ }^{c}$ PSL Research University, Chimie ParisTech - CNRS, Institut de Recherche de Chimie Paris, 75005, Paris, France. E-mail : regis.gauvin@chimieparistech.psl.eu

\section{Supporting information}

\section{Table of Contents}

Figure S1: Conversion and selectivity of $\mathrm{MO}$ ethenolysis with $(\equiv \mathrm{SiO})_{2} \mathrm{~W}(=\mathrm{O}) \mathrm{Ns}_{2} \quad \mathrm{~S} 2$

Table S1: Effect of the temperature on MO ethenolysis with $(\equiv \mathrm{SiO})_{2} \mathrm{~W}(=\mathrm{O}) \mathrm{Ns}_{2} \quad \mathrm{~S} 2$

Figure S2: Conversion and selectivity of $\mathrm{MO}$ ethenolysis with $(\equiv \mathrm{SiO})_{2} \mathrm{~W}(=\mathrm{O}) \mathrm{Ns}_{2}$ at various initial $\mathrm{S} 3$ ethylene pressure

Figure S3: Conversion and selectivity of $\mathrm{MO}$ ethenolysis with $(\equiv \mathrm{SiO})_{2} \mathrm{~W}(=\mathrm{O}) \mathrm{Ns}_{2}$ at various initial $\mathrm{S} 3$ ethylene pressure

Figure $\mathrm{S} 4$ : Conversion and selectivity of $\mathrm{MO}$ ethenolysis with $(\equiv \mathrm{SiO})_{2} \mathrm{Mo}(=\mathrm{O}) \mathrm{Np}_{2}$ at constant ethylene $\mathrm{S} 3$ pressure

Figure S5: Conversion and selectivity of 9-octadecene ethenolysis with $(\equiv \mathrm{SiO})_{2} \mathrm{Mo}(=\mathrm{O}) \mathrm{Np}_{2}$

Figure S6: Effect of the MO/Mo ratio on the conversion and selectivity of MO ethenolysis with S4 $\mathrm{MoO}(\mathrm{Np})_{2} / \mathrm{SiO}_{2-200}$ 


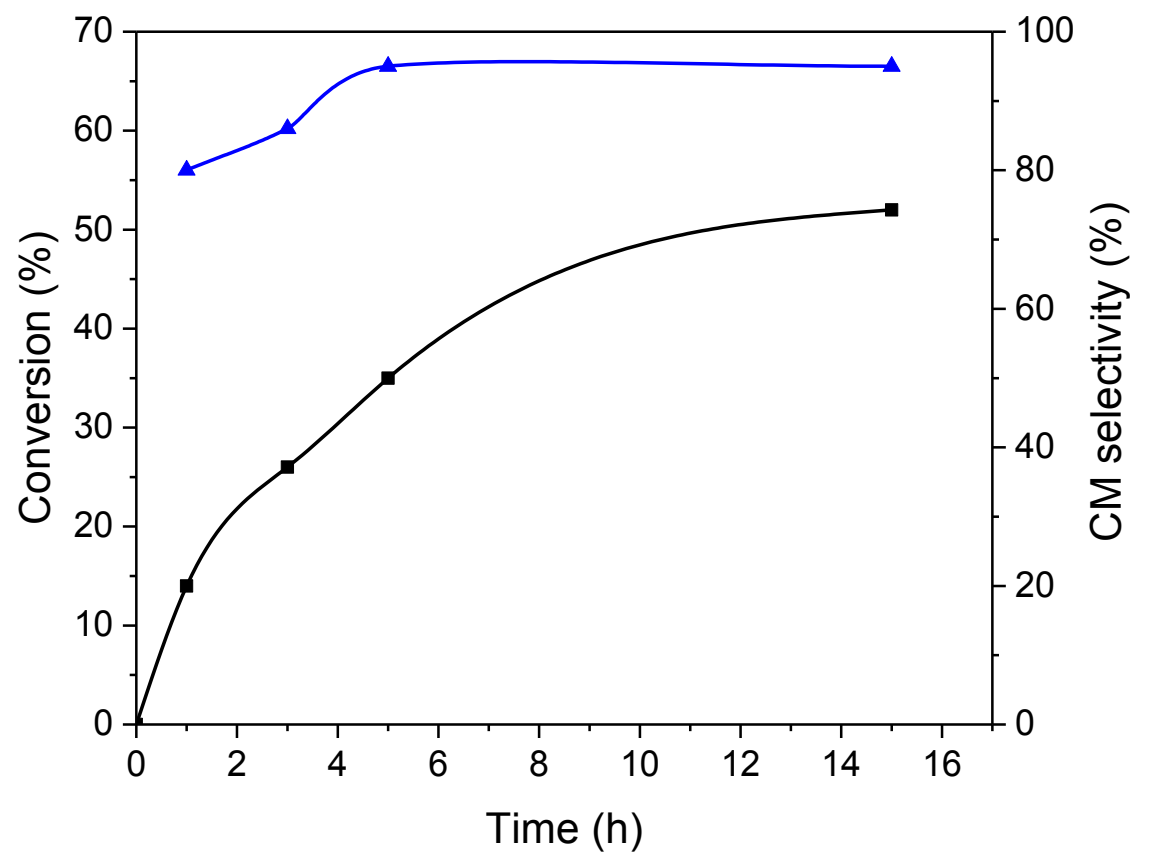

Figure S1: Conversion $(\boldsymbol{\square})$ and selectivity $(\boldsymbol{\Delta})$ of $\mathrm{MO}$ ethenolysis with $(\equiv \mathrm{SiO})_{2} \mathrm{~W}(=\mathrm{O}) \mathrm{Ns}_{2}$ at $\mathrm{MO} / \mathrm{W}=1000,100$ ${ }^{\circ} \mathrm{C}$; initial $\mathrm{P}_{\mathrm{C} 2 \mathrm{H} 4}=10$ bar

Table S1: Effect of the temperature

\begin{tabular}{|c|c|c|c|c|c|c|c|c|}
\hline entry & catalyst & $\begin{array}{c}\text { MO:W } \\
\text { ratio }\end{array}$ & $\begin{array}{c}\text { Temperature } \\
\left({ }^{\circ} \mathrm{C}\right)\end{array}$ & $\begin{array}{c}\text { Pression } \\
\text { (Bar) }\end{array}$ & time (h) & conv. $(\%)$ & $\begin{array}{c}\text { CM Sel. } \\
(\%)^{\mathrm{a}}\end{array}$ & TON \\
\hline 1 & $(\equiv \mathrm{SiO})_{2} \mathrm{~W}(=\mathrm{O}) \mathrm{Ns}_{2}$ & 1000 & 100 & 10 & 1 & 14 & 80 & 140 \\
\hline 2 & $(\equiv \mathrm{SiO})_{2} \mathrm{~W}(=\mathrm{O}) \mathrm{Ns}_{2}$ & 1000 & 100 & 10 & 3 & 26 & 86 & 520 \\
\hline 3 & $(\equiv \mathrm{SiO})_{2} \mathrm{~W}(=\mathrm{O}) \mathrm{Ns}_{2}$ & 1000 & 100 & 10 & 5 & 35 & 95 & 350 \\
\hline 4 & $(\equiv \mathrm{SiO})_{2} \mathrm{~W}(=\mathrm{O}) \mathrm{Ns}_{2}$ & 1000 & 100 & 10 & 15 & 52 & 95 & 520 \\
\hline 5 & $(\equiv \mathrm{SiO})_{2} \mathrm{~W}(=\mathrm{O}) \mathrm{Ns}_{2}$ & 1000 & 150 & 10 & 1 & 43 & 95 & 430 \\
\hline 6 & $(\equiv \mathrm{SiO})_{2} \mathrm{~W}(=\mathrm{O}) \mathrm{Ns}_{2}$ & 1000 & 150 & 10 & 3 & 45 & 94 & 450 \\
\hline 7 & $(\equiv \mathrm{SiO})_{2} \mathrm{~W}(=\mathrm{O}) \mathrm{Ns}_{2}$ & 1000 & 150 & 10 & 5 & 47 & 92 & 470 \\
\hline 8 & $(\equiv \mathrm{SiO})_{2} \mathrm{~W}(=\mathrm{O}) \mathrm{Ns}_{2}$ & 1000 & 200 & 10 & 1 & 50 & 94 & 500 \\
\hline 9 & $(\equiv \mathrm{SiO})_{2} \mathrm{~W}(=\mathrm{O}) \mathrm{Ns}_{2}$ & 1000 & 200 & 10 & 3 & 52 & 95 & 520 \\
\hline 10 & $(\equiv \mathrm{SiO})_{2} \mathrm{~W}(=\mathrm{O}) \mathrm{Ns}_{2}$ & 1000 & 200 & 10 & 5 & 54 & 94 & 540 \\
\hline
\end{tabular}

MO ethenolysis at 100,150 and $200{ }^{\circ} \mathrm{C}\left(\mathrm{MO} / \mathrm{W}=1000\right.$; initial $\mathrm{P}_{\mathrm{C} 2 \mathrm{H} 4}=10$ bar $),{ }^{\text {a }}$ selectivity in cross-metathesis. 

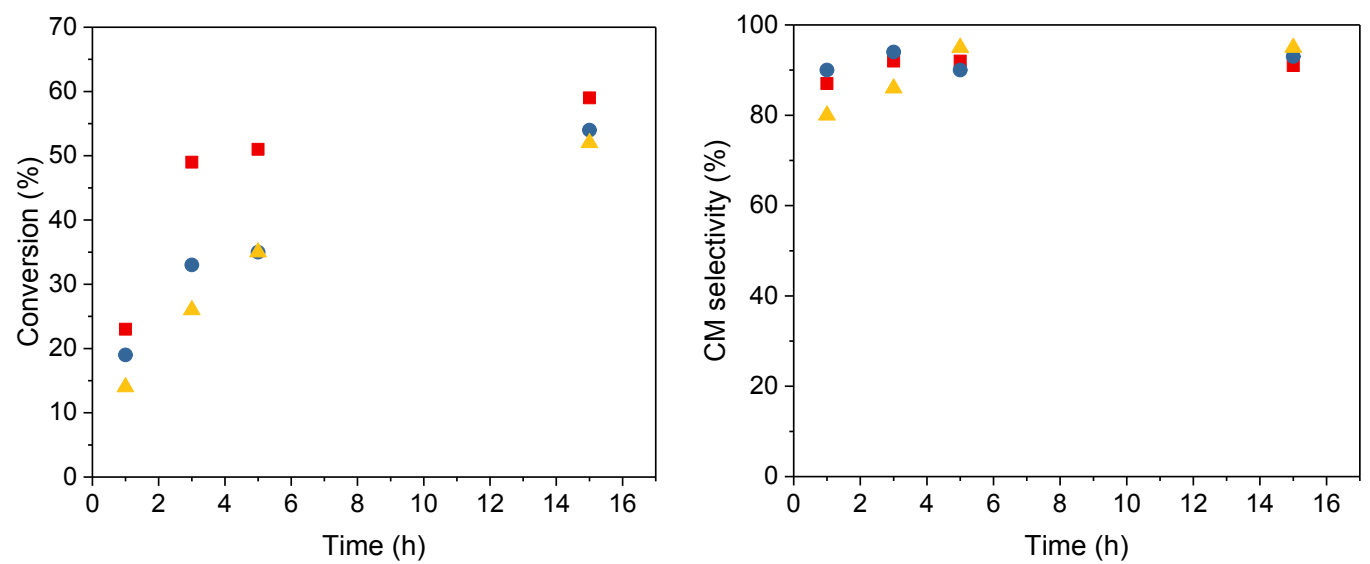

Figure S2: Conversion (left) and selectivity (right) of $\mathrm{MO}$ ethenolysis with $(=\mathrm{SiO})_{2} \mathrm{~W}(=\mathrm{O}) \mathrm{Ns}_{2}$ at initial ethylene pressure $=2(\boldsymbol{\bullet}), 5(\bullet)$ and $10 \operatorname{bar}(\boldsymbol{\Delta})\left(\mathrm{MO} / \mathrm{W}=1000 ; \mathrm{T}=100^{\circ} \mathrm{C}\right)$
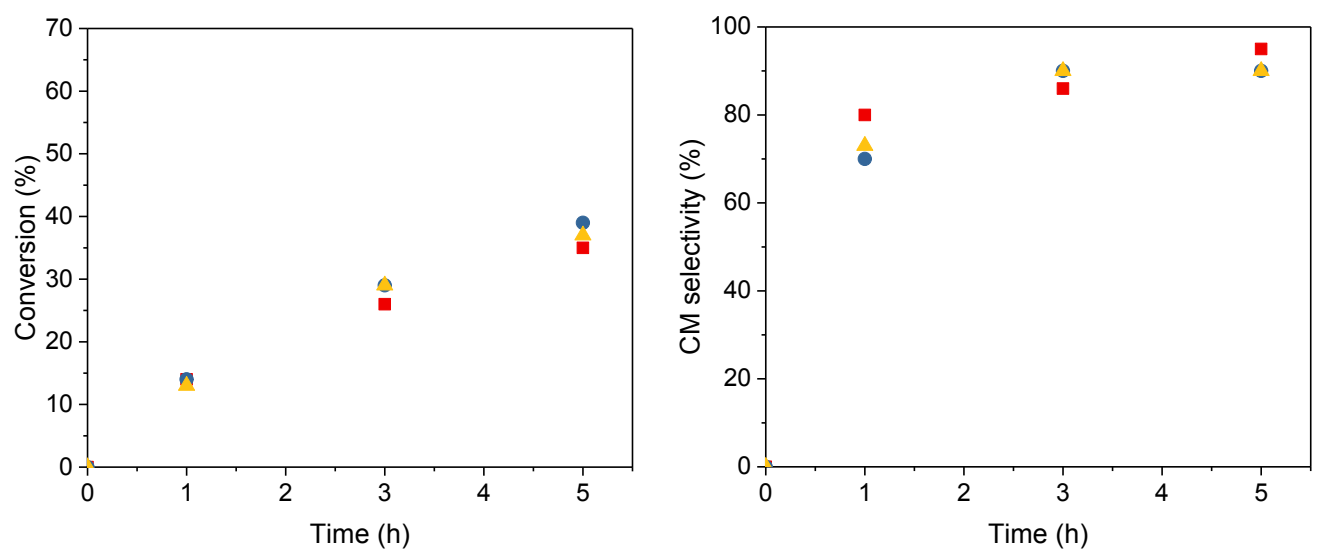

Figure S3: Conversion (left) and selectivity (right) of $\mathrm{MO}$ ethenolysis with $(\equiv \mathrm{SiO})_{2} \mathrm{~W}(=\mathrm{O}) \mathrm{Ns}_{2}$ at initial ethylene pressure $=10(\bullet), 20(\bullet)$ and $30 \operatorname{bar}(\mathbf{\Delta})\left(\mathrm{MO} / \mathrm{W}=1000 ; \mathrm{T}=100^{\circ} \mathrm{C}\right)$
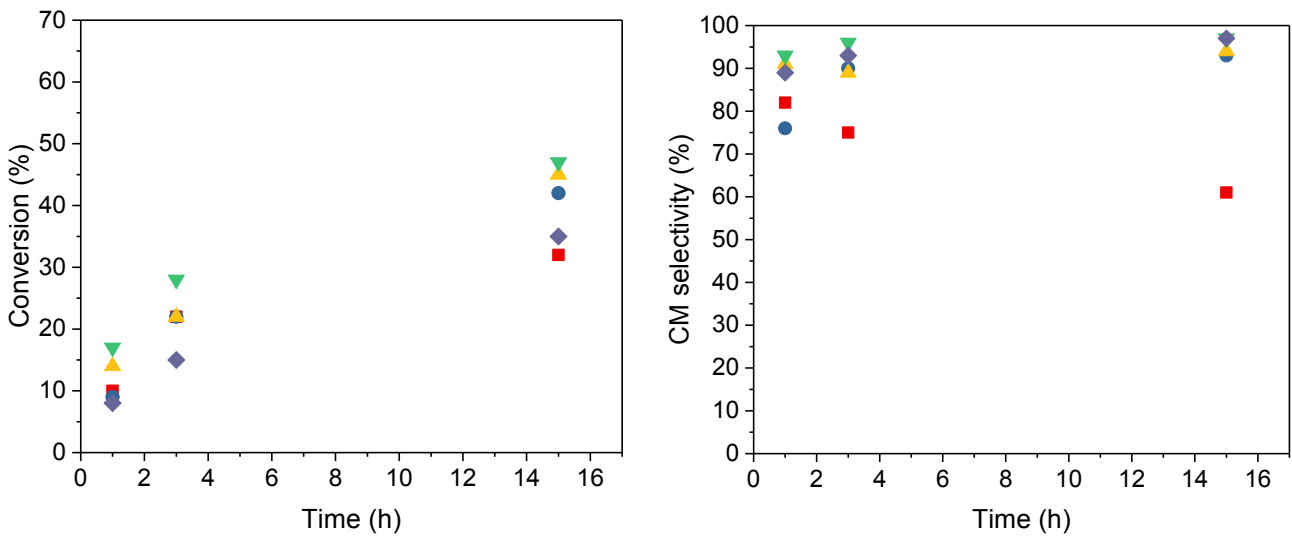

Figure S4: Conversion (left) and selectivity (right) of $\mathrm{MO}$ ethenolysis with $(\equiv \mathrm{SiO})_{2} \mathrm{Mo}(=\mathrm{O}) \mathrm{Np}_{2}$ at constant ethylene pressure $=0.5(\bullet), 1(\bullet), 2(\boldsymbol{\Delta}), 5(\boldsymbol{\nabla})$ and $10 \mathrm{bar}(\bullet)\left(\mathrm{MO} / \mathrm{W}=1000 ; \mathrm{T}=100{ }^{\circ} \mathrm{C}\right)$ 


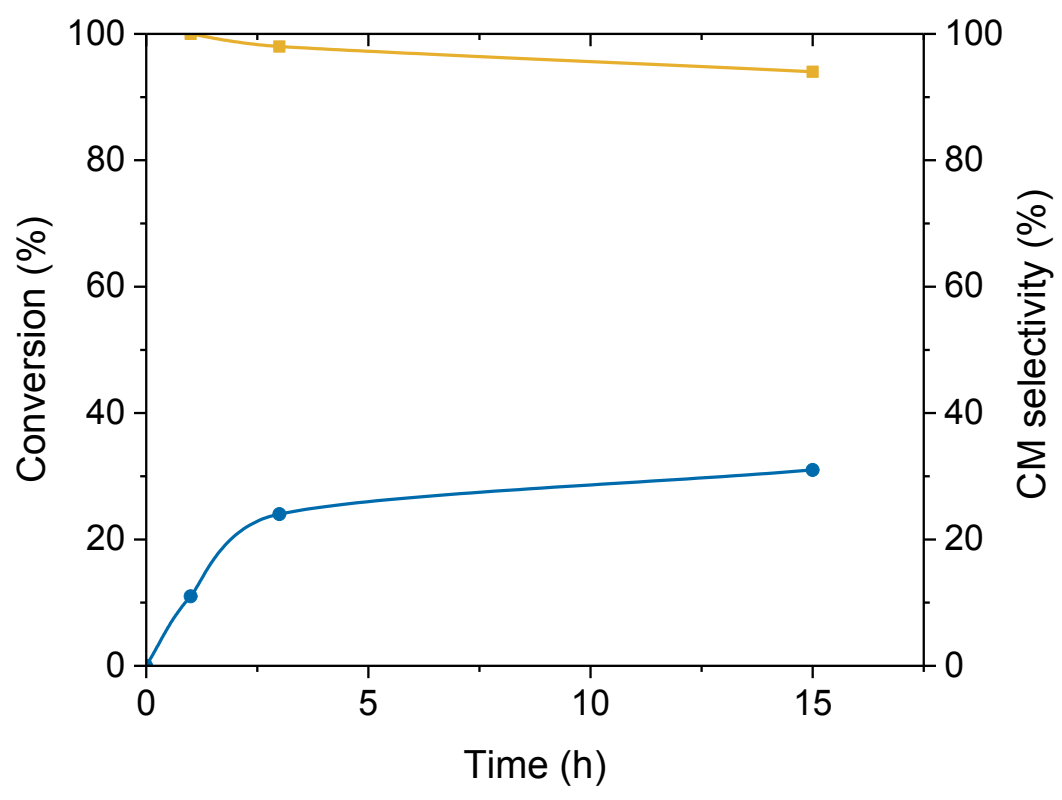

Figure S5: Conversion $(\bullet)$ and selectivity $(\bullet)$ of 9-octadecene ethenolysis with $(\equiv \mathrm{SiO})_{2} \mathrm{Mo}(=\mathrm{O}) \mathrm{Np}_{2}(9-$ octadecene $/ \mathrm{W}=1000,100^{\circ} \mathrm{C}$, constant ethylene pressure of 5 bar)
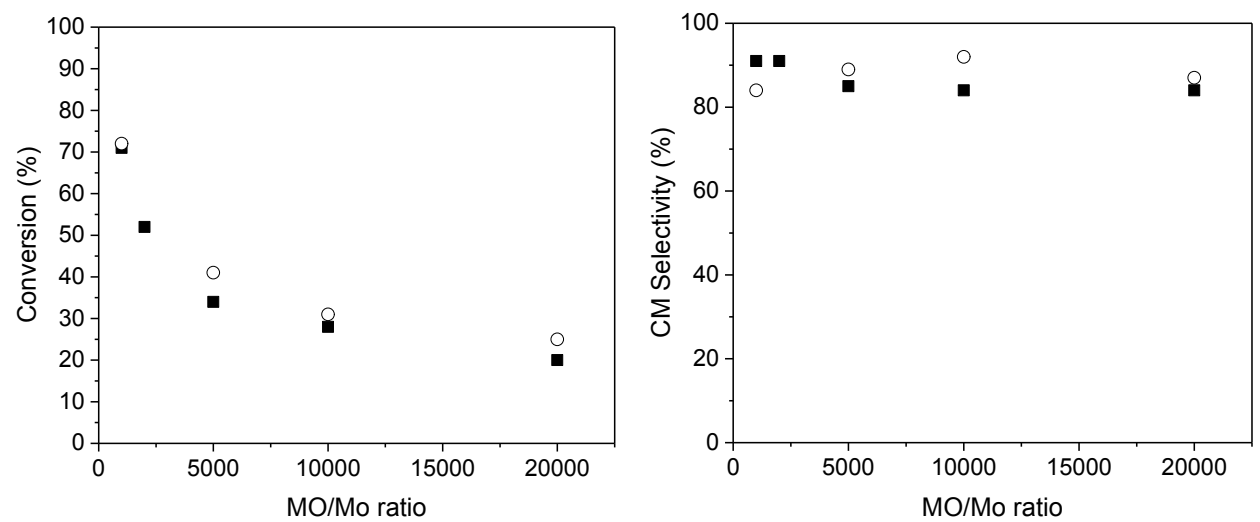

Figure S6: Effect of the MO/Mo ratio on the conversion and selectivity of MO ethenolysis with $\mathrm{MoO}(\mathrm{Np})_{2} / \mathrm{SiO}_{2-200}$ after $15 \mathrm{~h}$ reaction $50{ }^{\circ} \mathrm{C}$, constant ethylene pressure of $2(\circ)$ and 5 bar (๘)) 